\title{
A Sensitive Neutron Counter with a High-Pressure Ionization Chamber Filled with $\mathbf{B F}_{3}$
}

\author{
Institute of Atomic Energy, 05-400 Otwock-Świerk, Poland \\ e-mail: e01nat@cyf.gov.pl
}

\begin{abstract}
A ionization chamber, filled with ${ }^{10} \mathrm{BF}_{3}$ and operating in a pulse mode, was investigated as a possible sensitive pocket detector for detection of neutrons at low flux densities. The chamber is 85 $\mathrm{mm}$ long and $19 \mathrm{~mm}$ in diameter. The measured sensitivity of the chamber placed near the human body (in the belt), without any additional moderator was of the order of $1 \mathrm{pulse} / \mathrm{n} \mathrm{cm}^{-2}$ in the radiation field of a ${ }^{252} \mathrm{Cf}$ source.
\end{abstract}

Key words: neutron counter, ionization chamber, $\mathrm{BF}_{3}$.

\section{Introduction}

New demand appeared recently for the detection of neutrons at low flux densities in order to prevent possible smuggling of nuclear fissile materials. The aim of this work was to design a rugged, inexpensive neutron detector, small and simple enough to be used as a pocket device by the police and customs officers.

The main requirements for the pocket instrument are high sensitivity and reliability, while the requirements of accuracy of measurements, of the resolution time of the detector and of flatness of its energy response are not excessive. Pocket detectors should be, obviously, small, not too heavy and user-friendly.

The most frequently used neutron detectors are proportional counters, filled with either ${ }^{3} \mathrm{He}$ or gas compounds made with ${ }^{10} \mathrm{BF}_{3}$. The technology of manufacturing such devices for laboratory use and field measurements is well established, but there are 
several disadvantages of proportional counters when a pocket detector is to be designed. Since proportional counters use gas multiplication, their detection signals are highly sensitive to gas impurities. Thus, the gas in a proportional-counter tube must be at least 99.999 percent pure. This considerably increases the price of the detector. Moreover, the proportional counter's wire electrode can easily be made to vibrate, thereby producing spurious signals. In general, proportional detectors are susceptible to shock and vibration.

We propose here to use a ionization chamber, which is a detector operating without any gas amplification. Commercially available, inexpensive microchips (low noise sensitive charge amplifiers) can amplify charge pulses without gas multiplication, making it possible to use ionization chambers working in a pulse mode.

The chamber investigated in this work was filled with ${ }^{10} \mathrm{BF}_{3}$, but also $\mathrm{BF}_{3}$ with natural isotope composition can be used. The choice of the gas was, first of all, determined by its low price, but also by the relatively high energy of charged particles generated in the gas cavity by thermal neutrons. The neutron-absorbing nuclear reaction $\mathrm{n}+{ }^{10} \mathrm{~B} \rightarrow{ }^{7} \mathrm{Li}+\alpha$ results in the generation of two charged particles with a total energy of $2.31 \mathrm{MeV}$, which is considerably higher than the proton energy from the $\mathrm{n}+{ }^{3} \mathrm{He} \rightarrow{ }^{3} \mathrm{~T}+\mathrm{p}$ reaction. Therefore, the electronic amplification of the electrical charge and discrimination of pulses from gamma radiation is easier in the case of $\mathrm{BF}_{3}$. Another possible option would be to use a ${ }^{6} \mathrm{Li}$ layer on the chamber electrodes. The energy liberated in the nuclear reaction $\mathrm{n}+{ }^{6} \mathrm{Li} \rightarrow{ }^{3} \mathrm{~T}+\alpha$ is even higher $(4.78 \mathrm{MeV})$, but the cross section for the reaction with ${ }^{6} \mathrm{Li}$ is about four times lower than that for the reaction with ${ }^{10} \mathrm{~B}$. Moreover, ${ }^{6} \mathrm{Li}$ is not an easily available material.

\section{Ionization Chamber}

A special, aluminium-walled ionization chamber operating in a pulse mode has been designed. The chamber is $85 \mathrm{~mm}$ long and $19 \mathrm{~mm}$ in diameter. A $0.3 \mathrm{~mm}$ thick aluminium housing of the chamber was connected as one of the electrodes (at zero potential). The second, central electrode was made from a copper rod, $1.5 \mathrm{~mm}$ in diameter. 


\section{Measurements}

The chamber was investigated in reference radiation fields of ${ }^{239} \mathrm{Pu}-\mathrm{Be}$ and ${ }^{252} \mathrm{Cf}$ in calibration laboratory of the IAE Swierk and in a gamma radiation field of ${ }^{137} \mathrm{Cs}$.

Most of the measurements were made using the chamber placed in a spherical paraffin moderator with the wall thickness of about $100 \mathrm{~mm}$.

The chamber was filled with ${ }^{10} \mathrm{BF}_{3}$ up to about $150 \mathrm{kPa}$, which was the optimum gas pressure determined for a neutron radiation field. At higher gas pressures, ion recombination in the gas causes considerable decrease in the chamber sensitivity (see the dependence of the chamber sensitivity on gas pressure in Figure 1.)

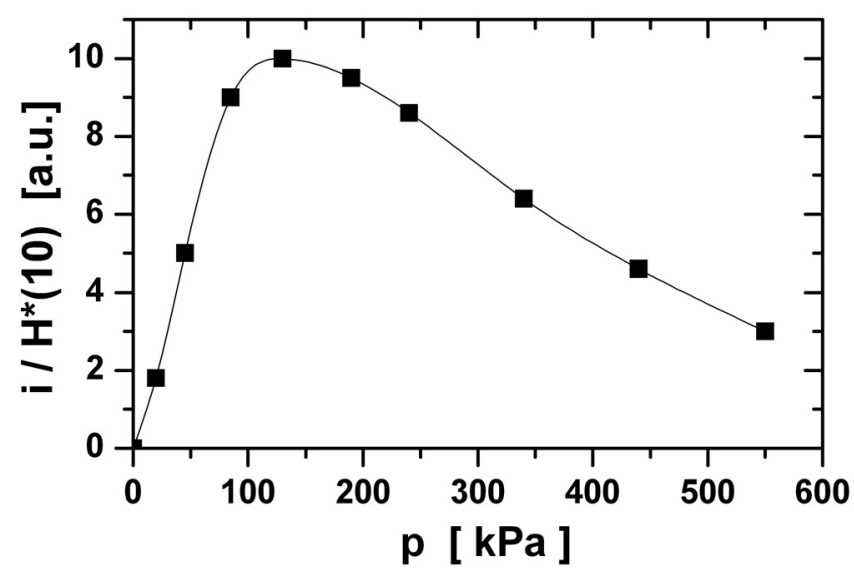

Figure 1. Dependence of the chamber sensitivity on the gas pressure for a ${ }^{239} \mathrm{Pu}$-Be source.

\section{Results}

The example of the discrimination curve of the chamber (the total number of counted pulses versus the discrimination level) and the pulse height distribution are shown in Figure 2 for the chamber polarized with the voltage of $600 \mathrm{~V}$. The measurements were carried out at a distance of $1 \mathrm{~m}$ from the ${ }^{239} \mathrm{Pu}$-Be source with a neutron emission rate of $2.79 ? 10^{7} \mathrm{n} / \mathrm{s}$. The noise of the device and its electronics (the signal measured without the radiation source) is also displayed as a dashed curve. 


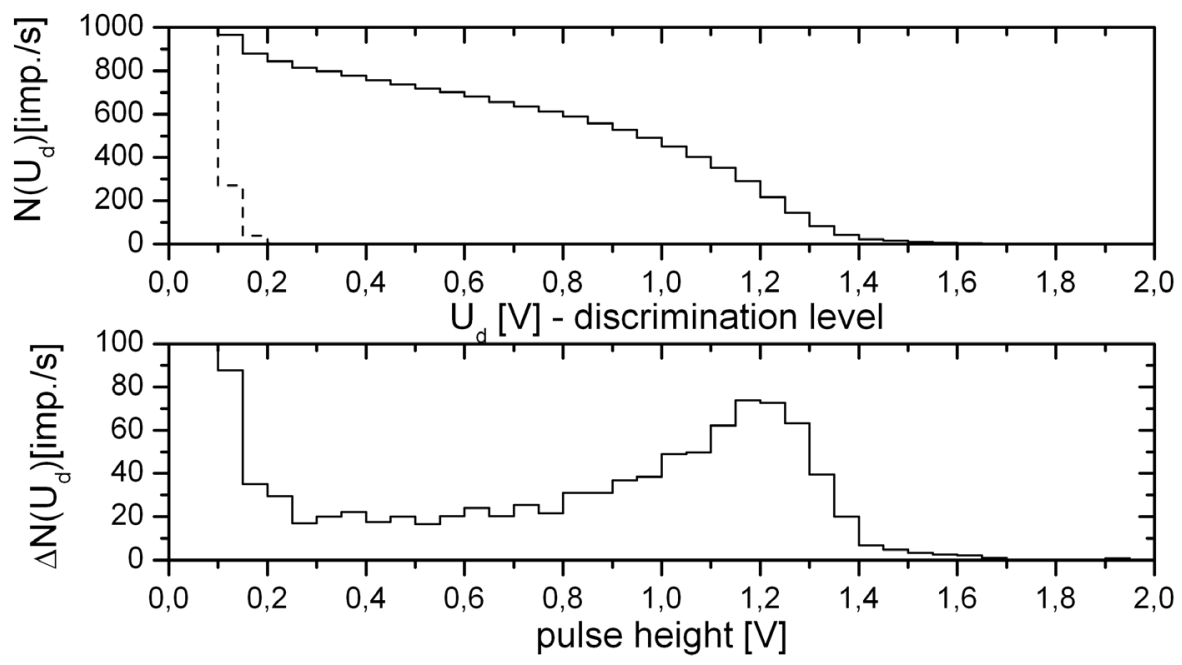

Figure 2. The discrimination curve and the pulse height distribution for the chamber operating at $600 \mathrm{~V}$. The dashed curve represents the signal measured without a radiation source (noise).

The pulse height distribution exhibits a well-pronounced maximum at $1.125 \mathrm{~V}$. The lower pulses between $0.3 \mathrm{~V}$ and about $0.6 \mathrm{~V}$ can be related to the events when the charged particles did not stop in the gas or when considerable recombination of ions occurred in the chamber. The range of alpha particles created in the gas is about $5 \mathrm{~mm}$, so it is comparable with the distance between the electrodes $(8.5 \mathrm{~mm})$.

The sensitivity of the chamber to ${ }^{239} \mathrm{Pu}$-Be and ${ }^{252} \mathrm{Cf}$ neutrons, is defined here as the ratio of the total number of counted pulses to the neutron ambient dose equivalent, $\left.H^{*}(10)\right)$. The dependence of the chamber sensitivity on the moderator thickness is shown in Figure 3. For the $100 \mathrm{~mm}$ thick moderator, the chamber sensitivity is of about 2500 pulses per $\mu \mathrm{Sv}$, when the discrimination level is set at $0.4 \mathrm{~V}$. This value is about twice as high as the sensitivity of a common Studsvik-Alnor 2202D remmeter which has a moderator of a similar size.

The measurements in a ${ }^{137} \mathrm{Cs}$ radiation field have shown that the chamber operating in a pulse mode with the discrimination level of $0.4 \mathrm{~V}$, was not sensitive to gamma radiation, at least up to $100 \mathrm{mSv} / \mathrm{h}$. 


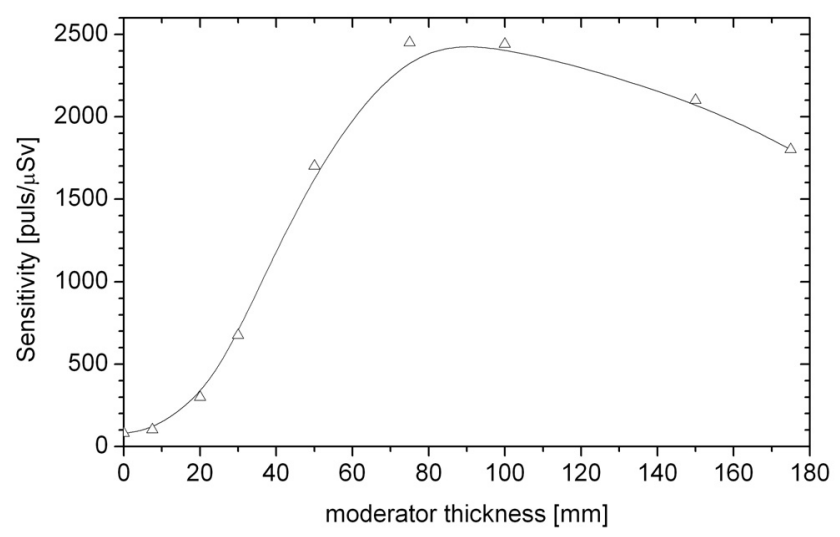

Figure 3. Dependence of the chamber sensitivity on the paraffin moderator thickness for ${ }^{252} \mathrm{Cf}$ source.

It can be seen that a relatively large volume of the moderating material is needed in order to ensure the optimum sensitivity of the chamber. Such a volume is definitely too large for the pocket detector. The problem can be at least partly solved when the chamber is attached close to the human body (with a belt), without any additional moderator. The measured sensitivity of the chamber operating under such conditions was of the order of 1 pulse $/ \mathrm{n} \mathrm{cm}^{-2}$ (800 pulses per $\mu \mathrm{Sv}$ ) in the radiation field of ${ }^{252} \mathrm{Cf}$.

\section{Conclusions}

A ionization chamber filled with $\mathrm{BF}_{3}$ can operate in a pulse mode at elevated gas pressures - in our case of about $150 \mathrm{kPa}$. Natural $\mathrm{BF}_{3}$ (not enriched in ${ }^{10} \mathrm{~B}$ ) can be used. The sensitivity of the chamber is sufficiently high for pocket detectors. The chamber does not require very high purity of the filling gas, and a rather broad range of polarizing voltages can be used, without considerable change in the chamber sensitivity. In our case, the minimum polarizing voltage was about $300 \mathrm{~V}$. Practically, any shape of electrodes can be used. The design and manufacturing technology can be simpler than the technology needed for proportional counters with stable gas amplification. Moreover, ionization chambers are usually more resistant to shocks and to harsh environmental conditions. 
The use of higher pressures of $\mathrm{BF}_{3}$ is possible. Therefore, the detectors with smaller thickness can be designed ensuring the same sensitivity as that measured in this work.

The chamber can operate both in pulse and current modes. This can ensure a very broad dynamic range for detection of neutrons. Also gamma radiation can be detected in the current mode. 